\title{
Avaliação qualitativa do hábito de leitura e entendimento: recepção das informações de produtos alimentícios
}

\author{
Qualitative evaluation of the reading habit and understanding: \\ reception of the information contained in labels of food products
}

\author{
Bianca Ramos MARINS ${ }^{1 *}$, Silvana do Couto JACOB ${ }^{1}$, Frederico PERES ${ }^{2}$
}

\begin{abstract}
Resumo
A rotulagem de alimentos é o principal elo entre o consumidor e o produto, além de representar um importante instrumento de educação alimentar. O presente estudo avaliou o hábito de leitura e compreensão dos rótulos de produtos alimentícios pelos freqüentadores de supermercados do município de Niterói/RJ - nos meses de setembro a dezembro de 2003. Foram realizadas entrevistas individuais (estudo quali-quantitativo) nas quais foi usado como instrumento de estudo um questionário sobre os rótulos, que foi aplicado a 400 indivíduos. Os resultados referentes à parte qualitativa do estudo evidenciaram que os principais problemas na compreensão da rotulagem dos produtos alimentícios foram: a baixa confiança pelos consumidores nas informações vinculadas pelos rótulos; o uso da linguagem técnica; o excesso de propagandas veiculadas pelas diversas mídias; e a pouca informação sobre componentes alimentares potencialmente alergênicos. O estudo apontou a necessidade do desenvolvimento de políticas públicas de comunicação para melhor orientar os consumidores na escolha de seus alimentos através da rotulagem de alimentos, estimulando-os no desenvolvimento de práticas alimentares mais adequadas.

Palavras-chave: rotulagem de alimentos; consumidor; legislação; avaliação qualitativa; vigilância sanitária.
\end{abstract}

\begin{abstract}
The food label is an important link between consumers and products, and may be used for educational purposes. This study was carried out aiming at evaluating the habit of reading and understanding foodstuff label contents. The population studied was formed by clients of a supermarket chain located in the city of Niterói/RJ, Brazil - from September to December, 2003. Individual interviews were carried out (qualiquantitative study) in which a questionnaire about the labels was used and applied to 400 customers. The results regarding the qualitative part of the study showed that the main problems in the understanding of the food product labels are: the low confidence of the consumers in the information on the labels, the use of technical language, the excess of propaganda made by different means of communication and the scarce information on potentially allergenic food components. The study pointed out the need for the development of public communication strategies to better guide consumers to choose their food.
\end{abstract}

Keywords: food label; consumers; legislation; qualitative evaluation; sanitary surveillance.

\section{Introdução}

A saúde de um indivíduo depende de fatores como: estado nutricional, disponibilidade do alimento, além de condições de saneamento ambiental, renda, educação, salário, habitação, lazer, transporte, acesso aos serviços de saúde, segurança alimentar, enfim, de políticas públicas, sociais, econômicas, agrícolas, de educação e de saúde (ANGELIS, 1999).

A preocupação com os hábitos alimentares é crescente, e desde a década de 80 vem aumentando a conscientização do público a respeito da relação entre dieta e saúde (HICKMAN et al. apud CELESTE, 2001). Através de uma dieta equilibrada o organismo adquire a energia e os nutrientes necessários para o bom desempenho de suas funções e para a manutenção de um bom estado de saúde. De longa data, se conhece os prejuízos de- correntes quer do consumo alimentar insuficiente - deficiências nutricionais - quer do consumo alimentar excessivo - obesidade (MONDINI; MONTEIRO, 1994).

Recentes estudos comprovaram a estreita relação entre a composição dos alimentos de uma dieta e a ocorrência de enfermidades crônico-degenerativas, como as doenças cardiovasculares, o Diabetes mellitus, os diferentes tipos de câncer e a obesidade (SAUERBRONN, 2003).

De acordo com Couig (1993) apud Castro (2000), o conjunto de informações que são expressas nas embalagens dos alimentos pode ser útil como instrumento para prevenir problemas de saúde e, ao mesmo tempo, exercer um papel educativo na definição de hábitos alimentares.

Recebido para publicação em 6/3/2007

Aceito para publicação em 19/10/2007 (002353)

${ }^{1}$ Instituto Nacional de Controle de Qualidade em Saúde, Fundação Oswaldo Cruz, Av. Brasil, 4365, Manguinhos, CEP 21045-900, Rio de Janeiro - RJ, Brasil,

E-mail: biancarmarins@ig.com.br

2 Centro de Estudos da Saúde do Trabalhador e Ecologia Humana, Fundação Oswaldo Cruz, Av. Leopoldo Bulhões, 1480, Manguinhos, CEP 21045-900,

Rio de Janeiro - RJ, Brasil

${ }^{*}$ A quem a correspondência deve ser enviada 
Esta assertiva implica na necessidade de se entender as influências sociais, econômicas e culturais dos consumidores para se redefinir políticas públicas de educação e comunicação que sejam capazes de orientar e estimular os consumidores em relação a hábitos alimentares mais adequados. As informações do rótulo devem, obrigatoriamente, descrever as características do produto, de modo a elucidar o consumidor sobre o mesmo.

O aumento do intercâmbio comercial entre as nações, devido à criação de mercados comuns - nos quais os alimentos ocupam lugar de destaque - tem contribuído para o estabelecimento de padrões de qualidade e normas técnicas comuns, visando harmonizar o fluxo de mercadorias entre os países parceiros, em uma economia global (REIS JÚNIOR; BRANDÃO apud NUNES et al., 1998). No que diz respeito especificamente ao comércio de alimentos, o Codex Alimentarius, através do Joint FAO \WHO Food Standards Program, se evidencia como principal norma regulamentadora, adotada em escala global. O objetivo do Codex, entre outras coisas, é recomendar normas para padronização de alimentos, delineando especificações técnicas para produtos e práticas de produção, assegurando não apenas o livre movimento de bens, mas a garantia de proteção da saúde do consumidor (GOURLIE, 1995; TABAI, 2002).

A adoção das recomendações do Codex pelos países beneficia os consumidores, tanto nos aspectos relacionados à higiene e à composição nutricional do produto, quanto naqueles relacionados ao acesso a informações mais adequadas sobre os alimentos em questão (MIRILLI; RESENDE; MARTINELLI, 1996). Em 1965, a Comissão do Codex estabeleceu o Comitê sobre Rotulagem de Alimentos, pois reconheceu a rotulagem de alimentos como canal de informação entre a indústria produtora de alimentos e o consumidor. A partir desta data, observou-se nos países do então chamado Primeiro Mundo (Países Desenvolvidos) a adoção sistemática às normas e regulamentos estabelecidos por este Comitê (HURT, 2002; SHIMIZU, 2002). Já nos países do então chamado Terceiro Mundo (Países em Desenvolvimento), a adoção destas normas era menos observada, uma vez que as Leis nacionais de alguns destes países não previam a obrigatoriedade de uma série de procedimentos e padrões recomendados por aquele Comitê (TEE et al., 2002).

No Brasil, considera-se um marco na legislação de alimentos o Decreto Lei $\mathrm{n}^{\circ}$ 986, de 21 de outubro de 1969, ainda em vigência, que institui as Normas Básicas sobre Alimentos, cujo Capítulo III normatiza sobre os aspectos gerais da rotulagem de alimentos, com recomendações de ordem mais geral (BRASIL, 2004). Apesar da importância de tal decreto, mais de duas décadas se passaram sem a publicação de nenhuma outra norma relacionada à rotulagem de produtos alimentícios, o que certamente colocou o país em desvantagem quanto à comercialização de seus produtos alimentícios no exterior.

Somente em 1993, em função do comércio entre os países membros do bloco econômico do Cone Sul (Mercosul), o Governo Brasileiro publicou a Resolução n 36 de 1993, que aprovou os regulamentos técnicos para padronização da rotulagem de alimentos embalados, incluindo aqueles produtos de origem animal. Esta Resolução foi regulamentada, em nível nacional, pelo Ministério da Agricultura Pecuária e Abastecimento, através da Portaria n 371, de 04 de setembro de 1997 (MAPA, 2003) e, posteriormente, pela Agência Nacional de Vigilância Sanitária, através da RDC 259 de 20/09/2002 (ANVISA, 2004). De acordo com essa Portaria, tornaram-se obrigatórios: a apresentação da denominação de venda do alimento, a lista de ingredientes, o conteúdo líquido, a identificação da origem, a identificação do lote, a data de validade e as instruções sobre o preparo e uso do alimento, quando apropriado (LIMA; GUERRA; LIRA, 2003).

Estes marcos da legislação brasileira têm relevância no campo da saúde, visto que desde a adoção das normas técnicas internacionais para a rotulagem de alimentos, os rótulos destes produtos vêm sendo relacionados como um dos fatores de influência na determinação dos hábitos alimentares dos indivíduos (GOURLIE, 1995; LEVY; FEIN, 1998; MARIETTA; WELSHIMER; ANDERSON, 1999; ZARKIN, 1993). De acordo com estes autores, quando as informações disponibilizadas nos rótulos se apresentam de maneira clara e com precisão, $o$ consumidor tende a dar mais credibilidade ao produto e, em muitos casos, faz a opção por este produto na composição da dieta familiar. Através dos rótulos dos alimentos, o consumidor é capaz ainda de avaliar se o produto atende às suas necessidades nutricionais, sobretudo quando algum indivíduo da família é portador de alguma doença - relacionada com a alimentação - ou se está acima ou abaixo do peso desejado.

Assim, conhecer o nível de compreensão que os consumidores têm sobre os rótulos dos produtos disponíveis no mercado é mais que uma estratégia de análise de processo comunicativo, é o conhecimento de alguns dos determinantes da alimentação dos indivíduos e, por conseguinte, da situação da saúde dessas pessoas. O objetivo do presente trabalho foi analisar o hábito de leitura e o entendimento/recepção das informações contidas em rótulos de produtos alimentícios embalados, pela população adulta freqüentadora de uma rede de supermercados do município de Niterói/RJ.

\section{Material e métodos}

O presente trabalho compreendeu um estudo seccional realizado entre os meses de setembro e dezembro de 2003, através de uma abordagem quanti-qualitativa de investigação, baseada na aplicação de questionários estruturados a 400 informantes, selecionados aleatoriamente entre os consumidores de uma rede de supermercados do município de Niterói/RJ. Utilizou-se, como base, parte do questionário empregado por Coutinho e Monteiro (2002) para avaliar o uso e o entendimento da população adulta freqüentadora de supermercados do Plano Piloto - Distrito Federal, sobre a informação nutricional de alimentos e bebidas embalados.

A partir das modificações foi construída uma versão preliminar do instrumento de pesquisa baseado em Coutinho e Monteiro (2002) e outras literaturas científicas (FELIPE, 2003; NESTLE, 1998; NEUHOUSER; KRISTAL; PATTERSON, 1999; SLOAN, 2003) para melhor adequação das questões.

Posteriormente, o instrumento de estudo foi analisado quanto a: categorias e complexidade das questões; adaptação à 
realidade sócio-cultural brasileira e informações sobre validação (BABBIE, 2001).

Para avaliação do instrumento de pesquisa preliminar selecionou-se uma amostra para a realização das entrevistas - fase pré-teste. Esta foi constituída por 20 indivíduos profissionais do Instituto Nacional de Controle de Qualidade em Saúde (INCQS) com representantes de categorias distintas: professores-pesquisadores (4); alunos de pós-graduação (4); funcionários bolsistas de fontes de fomento diversas (2); servidores públicos (2); cargo em comissão (2); colaborador autônomo (2); terceirizado contratado (2); e terceirizado do serviço de limpeza (2). Utilizou-se como critério de inclusão, nesta pesquisa, os entrevistados maiores de 15 anos e alfabetizados. Não foram incluídos os indivíduos portadores de deficiências que pudessem dificultar a comunicação entre o entrevistado e o entrevistador.

Com base nas respostas e sugestões fornecidas, foram realizadas as alterações pertinentes na formulação das questões.

\section{1 Área geográfica e amostragem}

O município de Niterói pertence à Região Metropolitana do Rio de Janeiro. Limita-se ao norte com o município de São Gonçalo, ao leste com o município de Maricá, ao sul e a oeste com o Oceano Atlântico. Possui uma extensão total de $131,8 \mathrm{~km}^{2}$ e 48 bairros, distribuídos em cinco regiões de planejamento organizacional: Praias da Baía, Norte, Oceânica, Pendotiba e Leste. De acordo com os dados, o município tem aproximadamente 460 mil habitantes (IBGE, 2005).

Das cinco grandes redes de supermercado que têm estabelecimentos no município, apenas uma possui filiais em todas as regiões de planejamento, razão pela qual esta rede foi escolhida para o estudo. A seleção dos informantes se deu em obediência à lei de significância estatística e, para tanto, utilizou-se a Equação 1 adotada por Fonseca e Martins (1994):

$\mathrm{n}=\frac{\mathrm{z}^{2} \times \mathrm{p} \times \mathrm{q}}{\mathrm{i}^{2}}$

em que:

n = é o tamanho da amostra;

$\mathrm{Z}=$ corresponde à abscissa da curva normal padrão, fixado um nível de confiança (para 95\%, $z=1,96$ );

$\mathrm{p}=$ é o valor da estimativa da proporção;

$\mathrm{q}=$ é o valor de 1-p; e

$\mathrm{i}=$ é o erro amostral.

A amostra foi então definida em 400 informantes, distribuídos pelas filiais das cinco regiões administrativas através da observação do percentual de habitantes em cada região, estimado pelo IBGE (2005). Assim, de acordo com esta estimativa, os 400 informantes foram assim divididos: 167 na região das Praias da Baía; 136 na região Norte; 49 na região Oceânica; 43 na região de Pendotiba; e cinco na região Leste.

\subsection{Aplicação do instrumento de levantamento de dados}

A aplicação do questionário acontecia dentro de cada loja, tendo como local a entrada principal, localizada próximo ao guarda-volumes. Foram aplicados, no máximo, 10 questionários por dia, com duração média de 12 minutos cada. A escolha dos informantes foi aleatória, a partir do primeiro consumidor a passar pelo pesquisador, respeitando-se um intervalo de 2 minutos entre a aplicação de cada um dos questionários e o direito de recusa de cada entrevistado que, caso aceitasse participar, era informado sobre os aspectos éticos relacionados àquela pesquisa, em atenção à Resolução 196/96 do CNS.

Os questionários foram estruturados com perguntas fechadas, passíveis de quantificação, e abertas, de caráter subjetivo. As respostas dadas às perguntas abertas foram transcritas, integral e literalmente, no momento das entrevistas. Desta forma, foi possível obter um panorama geral da relação do consumidor com os rótulos dos alimentos embalados e, ao mesmo tempo, aprofundar questões de caráter qualitativo, relacionadas ao entendimento das informações contidas naqueles rótulos.

Em função da natureza subjetiva do tema, no presente trabalho discutiram-se apenas os resultados da análise qualitativa das respostas dadas às perguntas abertas do questionário, utilizando os demais dados de maneira pontual, apenas para caracterizar o grupo de respondentes da pesquisa.

\section{Resultados e discussão}

A análise dos dados obtidos com as respostas dadas às perguntas abertas do questionário mostrou uma série de problemas relacionados ao entendimento do conteúdo dos rótulos de alimentos embalados por parte dos consumidores entrevistados. Os principais problemas, que aqui serão discutidos, são aqueles que dizem respeito a: 1) relação entre leitura e entendimento dos conteúdos dos rótulos e a confiança no produto em questão; 2) relação entre o conteúdo do rótulo com as estratégias de propagandas das indústrias; 3) o uso de linguagem técnica (além de siglas, abreviaturas, etc.) nestes rótulos, dificultando o entendimento de seu conteúdo; e 4) o entendimento das informações sobre a presença de ingredientes possíveis de causar disfunções.

\subsection{Relação entre leitura e entendimento dos conteúdos dos rótulos e a confiança no produto}

Aproximadamente 24\% ( $\mathrm{n}=96)$ dos entrevistados informaram não confiar no conteúdo dos rótulos, seja por acreditarem que as informações ali contidas são manipuladas, omitidas ou falsas, seja por acharem que aquelas informações não sofrem fiscalização por parte dos órgãos competentes, como se pode observar nos relatos abaixo:

"Sei lá, não confio no homem, vou confiar em rótulo. Você confia em rótulo? Eu confio em Deus, no rótulo eles põem o que eles querem." (Vendedora, 41 a 60 anos)

"A fiscalização não é rigorosa, devia fiscalizar as empresas, fazer os cálculos certos e rigorosos pelo profissional de nutrição" (Nutricionista, 21 a 40 anos) 
Para o consumidor, a rotulagem é meramente figurativa, porque o mesmo acredita que quem determina o que deve ser descrito nos rótulos são as indústrias alimentícias que estariam interessadas em lucrar, informando ao consumidor somente o que lhe conviesse.

"Sei lá, as pessoas querem vender o produto e escrevem qualquer coisa." (Comerciante, 41 a 60 anos)

"Acho que é um cartel, eles combinam, eles controlam, eles fazem o que eles querem e ainda não há fiscalização, há corrupção." (Funcionário público, 41 a 60 anos)

"Porque são mentirosos, enganam na pesagem. Exemplo: tem produto que diz que tem $1 \mathrm{~kg}$ e não tem." (Costureira, 41 a 60 anos)

Segundo Quast (2002), as grandes indústrias de alimentos desenvolvem produtos de qualidade do ponto de vista sensorial e sanitário, mas elas não podem garantir com isso uma dieta adequada para todos, porque esta depende da escolha dos tipos e das quantidades de alimentos pelos consumidores. E, neste caso, o papel dos rótulos como determinante na escolha dos alimentos que serão consumidos é central. Ao desacreditarem nas informações contidas nos rótulos, até por reflexo do descrédito dado às indústrias (sobretudo às grandes corporações), o consumidor acaba por optar por outros produtos, recomendados por parentes e/ou aqueles que, desde muito tempo, fazem parte da composição de sua alimentação, tornando inútil grande parte do poder de informação dos rótulos:

\footnotetext{
"Eu confio naquilo em que eu compro de longa data, as informações dos rótulos pouco importam, eu gosto, e estou acostumada a comprar os alimentos de longa data, o que importa é o material." (Dona de casa, acima de 60 anos)
}

A garantia de uma dieta saudável realmente não pode ser alcançada sem que se tenha disponível uma quantidade mínima de informações sobre os constituintes desta dieta. E estas informações, de forma geral, são influenciadas por determinantes de ordem econômica, política e social, além de princípios e crenças individuais (NESTLE, 1998). O paradoxo que se apresenta aqui, e que será melhor discutido a seguir, pode ser entendido pela desconfiança do consumidor em relação às informações contidas nos rótulos de gêneros alimentícios, pois de acordo com os relatos dos entrevistados, quanto mais as indústrias investem em propaganda, maior é o sentimento de que aquelas informações não correspondem à realidade e são voltadas apenas a induzir a compra do produto pelo consumidor.

\subsection{Relação entre o conteúdo do rótulo com as estratégias de propagandas das indústrias}

Nos últimos anos, as estratégias de propaganda e venda desenvolvidas pelas indústrias produtoras de alimentos têm crescido assustadoramente, fortemente influenciadas por mercados consumidores em expansão nos mais diversos países, onde cada vez mais tem-se observado uma série de problemas de saúde decorrentes da alimentação inadequada das populações. De acordo com Pipetone (2005), atualmente é possível observar que as indústrias de alimentos investem muitos recursos com a veiculação de propagandas de seus produtos com o objetivo de conquistar a fidelidade de novos consumidores.

O consumo de refeições rápidas de elevado teor calórico (Fast Food), por exemplo, é uma verdadeira epidemia entre crianças e adolescentes, e encontra suas bases nas estratégias de venda (marketing) destes produtos. Parte da consistente relação entre assistir televisão e a obesidade nas crianças/adolescentes pode ser explicada pela forte e tentadora propaganda dos Fast Foods (ROBINSON, 1998). Elas são a grande maioria entre os anunciantes da categoria alimentação/restaurantes, perdendo apenas para a propaganda de redes de supermercado. As crianças e os adolescentes são freqüentemente os gruposalvo dos anunciantes destes produtos, devido à influência que exercem sobre os pais na hora da escolha dos alimentos a serem consumidos pela família (OMS, 1998).

São raras as estratégias educativas e de comunicação, sobre os riscos do consumo excessivo de produtos Fast Foods, voltadas para o segmento mais vulnerável à ação do marketing. Nesse sentido, é muito mais fácil associar as informações contidas nos rótulos dos alimentos com a propaganda do que relacioná-las às recomendações do Codex alimentarius.

Tais informações contribuíram para o entendimento dos relatos dos entrevistados, que associaram as informações dos rótulos a uma estratégia de venda da indústria:

"Quando começa a esticar muito dizer que tem propriedades assim ou assado, tem muito marketing para pouca coisa." (Repórter fotográfico, 41 a 60 anos)

"As coisas mais relevantes a ser destacadas, não misturar com propagandas e ser mais claro, as propagandas à parte.” (Engenheiro eletricista, 21 a 40 anos)

"Não confio no rótulo porque é propaganda." (Diarista, 41 a 60 anos)

E, como resultado, acabam por desacreditar por completo nas informações ali contidas:

\footnotetext{
“Acho besteira, (o rótulo) não tem função, não." (Auxiliar de colégio, 41 a 60 anos)

“Tudo caô.” (Mecânico, 21 a 40 anos)
}

Tendo em vista que a publicidade pode induzir e/ou ratificar tendências de consumo, Henriques, Ferreira e Dias (2006) ressaltam que a informação clara e adequada sobre os riscos do consumo exagerado de determinados produtos alimentícios pode contribuir para hábitos alimentares mais saudáveis, por isso considera-se necessário o desenvolvimento de políticas de segurança alimentar e nutricional que regulem a publicidade de alimentos. 


\section{$3.3 \mathrm{O}$ uso de linguagem técnica como siglas e abreviaturas dificultando o entendimento dos rótulos}

Tendo a rotulagem de alimentos a função inerente de informação, a prática da leitura deve ser exercida pelo consumidor no momento da aquisição do produto alimentício. Na maioria dos casos, a dificuldade de se adquirir o hábito da leitura é observada em relação à compreensão das informações dos rótulos pela população, esta dificuldade pode ser atribuída à utilização de uma linguagem técnica, cuja compreensão pode ser alcançada apenas por um público mais específico. Desta forma, também podemos observar uma contradição quanto à finalidade da rotulagem de alimentos, ou seja, ao mesmo tempo em que ela representa um elo entre o consumidor e o produto, só é decifrada por aqueles que estão mais capacitados; sejam eles com melhor conhecimento em relação às substâncias presentes ou em relação a outro idioma utilizado que não o português.

\footnotetext{
"Os nomes dos componentes são muito complicados, o que a gente não entende, nem as aulas de química resolvem, às vezes pensam que é por causa do nível de escolaridade e nem sempre é por isso." (Estudante, 15 a 20 anos)
}

Se por um lado, há uma defasagem em relação à compreensão dos rótulos dos produtos alimentícios pelos consumidores, fica constatada a necessidade de se desenvolver programas educativos para se difundir a informação, permitindo a comunicação de forma adequada entre consumidores e produtores de alimentos. Esta iniciativa, muitas vezes, deve ser mediada pelos órgãos governamentais, pois se por um lado há o interesse em se harmonizar as legislações com a finalidade de se minimizar as barreiras comerciais, por outro há a necessidade de se contextualizar, para a realidade da população brasileira, a linguagem utilizada, proibindo a utilização de vocábulos em língua estrangeira, abreviações e utilização de códigos que ocultem a identidade do aditivo:

"Não identifica o nome do conservante, do nome químico, usam apenas o código, tem a sigla, mas não sabe o nome daquele componente químico." (Enfermeira, 21 a 40 anos)

Deve-se buscar uma linguagem mais simples para a divulgação das informações e maior compreensão pela população. Esta linguagem não deve ser banal ou omissa, pois desta forma podem ocorrer distorções e interpretações errôneas das informações, conforme relato abaixo.

"Ser mais explicado, sem usar termos tão técnicos, exemplo carboidratos como que o povão vai saber o que é carboidratos, usar massas engordativas." (Enfermeira, 41 a 60 anos)

A utilização de uma linguagem pouco acessível à compreensão da maioria dos consumidores pode gerar desinteresse pelas informações dos rótulos ou até descrença. Estas situações talvez impliquem num descrédito por parte dos consumidores em mudanças que ocorram visando solucionar estes problemas.
Assim, as estratégias de educação e comunicação são medidas urgentes, podendo servir de estímulo aos consumidores para exercitarem o hábito de leitura dos rótulos. Por outro lado, a indústria de alimentos deve estar compromissada em cumprir os preceitos legais e informar ao consumidor as características inerentes ao produto.

Matta, Henriques e Silva (2006) evidenciaram que algumas indústrias ainda não estão adequadas à legislação, pois os dados obtidos pela avaliação da rotulagem de 84 produtos, entre eles barras de cereais e iogurtes, classificados como diet e light e comercializados no Rio de Janeiro, evidenciaram que 37,5\% da informação nutricional contida nos rótulos não apresentava cor em contraste com o fundo da embalagem; $2,1 \%$ não informava a data de validade e o lote; apenas $50 \%$ das barras de cereais diet informaram sobre o conteúdo de mono e de dissacarídeo para diabéticos; e nenhuma embalagem trazia informações sobre gordura trans e percentual de Valor Diário (VD) para 2000 kcal.

O desenvolvimento de qualquer iniciativa que vise auxiliar os consumidores na apropriação das informações dos rótulos torna-se inviabilizada se na rotulagem existem ou persistem lacunas, pois a informação dever ser suficientemente clara e capaz de esclarecer o consumidor. Entretanto, de acordo com Fiore (2006), que relatou a pesquisa realizada com cerca de 2200 adultos, constatou-se que a falta de noções básicas sobre nutrição compromete o entendimento sobre as informações dos rótulos, já que metade dos entrevistados não sabia quantas calorias deveria consumir diariamente e $80 \%$ dos entrevistados não sabiam quanto de gordura, carboidratos ou sódio deveria ser consumido em uma dieta de $2000 \mathrm{kcal}$.

"Não sei explicar o que, pois não tenho hábito de ler, é tanto nome complicado que a gente não entende." (Doméstica, 41 a 60 anos)

"As letras são muito miudinhas, dá a impressão que a gente está sendo passado para trás." (Doméstica, 21 a 40 anos)

A imperiosa necessidade de se estimular medidas educativas que possibilitem os consumidores a entender o significado das informações transmitidas pelos rótulos dos produtos permitirá ao consumidor selecionar os seus alimentos de forma mais consciente.

\footnotetext{
"Traduzir o científico para o popular, para as pessoas entenderem." (Médico, 41 a 60 anos)
}

\section{4 $O$ entendimento das informações sobre a presença de ingredientes que podem causar disfunções}

Muitas recomendações deveriam ser feitas usando-se termos simples para identificar a maioria dos alergênicos na listagem de ingredientes que devem ser destacados nos rótulos dos produtos alimentícios. Na rotulagem devem sempre constar informações em destaque no painel principal e que sirvam de precaução para os consumidores. É inadmissível que estes alergênicos alimentares não sejam declarados pelas indústrias produtoras de alimentos (WOOD, 2002). 
"Eles usam certos termos que não exemplificam ou explicitam, por exemplo, amido; amido de que; pois para mim é importantíssimo, pois tenho doença celíaca, eu não posso com amido de trigo, aveia, e cevada." (Engenheira, 41 a 60 anos)

“Tem uns nomes malucos, os nomes químicos eu não sei o que é em que quantidade desse elemento e se essa dosagem influencia ou não para uma alergia." (Dona de casa, 41 a 60 anos)

Consumidores com restrições alimentares são grupos que requerem uma atenção maior das autoridades em termos de fiscalização da produção e controle dos alimentos. Ao fabricante cabe a responsabilidade por qualquer omissão que possa levar danos à saúde do consumidor.

A presença de Organismos Geneticamente Modificados (OGMs) é uma questão que inspira cuidados, devido a vários fatores a eles associados e relacionados com a segurança para a saúde do consumidor. Estudos devem ser realizados a fim de avaliar os riscos suscetíveis, principalmente, à saúde de determinados grupos de consumidores que sofrem de alergias, e até as questões de ordem ética e religiosa.

Quanto ao resultado da última reunião do Comitê do Codex Alimentarius sobre a rotulagem de alimentos oriundos das modernas técnicas de biotecnologia, foi possível observar dois opositores; de um lado, a delegação dos Estados Unidos que defende a necessidade da rotulagem apenas se houver mudanças significativas na composição do produto, características, valor nutricional ou uso final do produto. E de outro, a delegação da União Européia que defende o propósito da rotulagem de munir o consumidor com informações úteis e não apenas atrair a sua atenção para informações de saúde e segurança (CONOVER, 2004).

Acredita-se que o referencial para qualquer discussão sobre a rotulagem de alimentos, sejam eles derivados ou não de modernas tecnologias, deve primar em obedecer aos anseios do consumidor, conforme o relato abaixo. O direito à informação é garantido em lei, pois a ela cabe decidir ou não sobre a aquisição de um produto ou serviço.

"Dizer os componentes transgênicos bem visível, ressaltar, pois o consumidor tem direito de saber. Eu, por exemplo, não gosto de transgênicos, não acho bom." (Repórter fotográfico, 41 a 60 anos)

\section{Conclusões}

Os rótulos representam um espaço de informação ao consumidor, e quando bem compreendidos permitem que as escolhas alimentares ocorram de forma mais criteriosa. Para que a rotulagem exerça o papel que lhe é inerente, as informações disponibilizadas devem ser fidedignas, legíveis e acessíveis a todos os segmentos sociais.

Os resultados obtidos no presente estudo possibilitaram as seguintes conclusões:
- Freqüentemente os rótulos dos produtos alimentícios geram dúvidas, descrédito ou insatisfação em relação às informações; e

- O excesso de propagandas, a utilização de linguagem técnica/abreviaturas/siglas, a falta de explicitação em relação aos componentes potencialmente alergênicos a grupos populacionais específicos, e o uso de letras pouco legíveis são fatores que dificultam a compreensão pelos consumidores.

Os organismos legisladores devem estabelecer os padrões técnicos para que os alimentos oferecidos à população estejam dentro de condições higiênico-sanitárias adequadas, cabendo aos produtores e comerciantes de alimentos o cumprimento dos preceitos legais.

É evidente a necessidade do desenvolvimento de políticas públicas na área de educação e comunicação, visando auxiliar os consumidores no processo de apropriação das informações vinculadas pelos rótulos dos produtos alimentícios.

\section{Agradecimentos}

À Fundação de Amparo à Pesquisa do Estado do Rio de Janeiro (FAPERJ), como órgão de fomento; aos Diretores da Rede de Supermercados onde o estudo foi realizado; ao Programa de Pós-Graduação em Vigilância Sanitária de Produtos do INCQS/FIOCRUZ; ao Rodrigo Costa Martins pela confecção do banco de dados; ao professor Licínio Esmeraldo da Silva do Instituto de Matemática do Departamento de Estatística da Universidade Federal Fluminense, pela avaliação do cálculo amostral e ao Armando Meyer, MSc do CESTEH/FIOCRUZ pelas avaliações estatísticas.

\section{Referências bibliográficas}

ANVISA - AGÊNCIA NACIONAL DE VIGILÂNCIA SANITÁRIA. RDC $n^{\circ} 259$, de 20 de setembro de 2002. Aprova o regulamento técnico sobre rotulagem de alimentos embalados. Disponível em: $<$ http://www.anvisa.gov.br>. Acesso em: 04 jun. 2004.

ANGELIS, R. C. Fome oculta: impacto para a população do Brasil. São Paulo: Atheneu, 1999.

BABBIE, E. Métodos de Pesquisa de Survey. Belo Horizonte: UFMG, 2001.

BRASIL. Decreto-lei no 986, de 21 de outubro de 1969. Institui normas básicas sobre alimentos. Disponível em: <http://www.anvisa.gov. br>. Acesso em: 10 mai. 2004.

CASTRO, V. M. F. Conteúdo informativo das embalagens de alimentos: um estudo exploratório internacional comparado, sob a ótica brasileira e norte-americana dos agentes de produção e consumo. São Paulo, 2000. Tese - (Doutorado em Saúde Pública), Faculdade de Saúde Publica, Universidade de São Paulo - USP.

CELESTE, R. K. Análise comparativa da legislação sobre rótulo alimentício do Brasil, Mercosul, Reino Unido e União Européia. Revista de Saúde Pública, v. 35, n. 3, p. 217-223, 2001.

CONOVER, R. V. Biotech Labeling still unresolved in Codex. Food Technology, v. 58, n. 6, p. 208, 2004.

COUTINHO J. G.; MONTEIRO R. A. Uso e entendimento pela população adulta freqüientadora de supermercados do Plano Piloto: Distrito Federal - da informação nutricional de alimentos 
e bebidas embalados. Brasília, 2002, 86p. Monografia - (Especialista em Saúde Coletiva), Faculdade de Ciências da Saúde, Departamento de Saúde Coletiva, Universidade de Brasília - UNB.

FELIPE, M. R. et al. Rotulagem de Alimentos: o comportamento do consumidor usuário de supermercados do Balneário Camboriú/SC. Higiene Alimentar, v. 17, n. 111, p. 49-57, 2003.

FIORE, P. Consumers want clarity in labeling. Food Technology, v. 60, n. 6, p. 136, 2006.

FONSECA, J. S.; MARTINS, G. A. Curso de Estatística. 5 ed. São Paulo: Atlas, 1994. cap. 7.

GOURLIE, K. E. Food labeling: a canadian and international perspectives. Nutrition Reviews, v. 53, n. 4, p. 103-107, 1995.

HENRIQUES, P.; FERREIRA, D. M.; DIAS, P. C. Regulamentação da propaganda de alimentos no Brasil: uma realidade em construção. Higiene Alimentar, v. 21, n. 150, p. 374-375, 2006.

HURT, E. Nutrition labelling: European Union and United Kingdom perspectives. Asia Pacific Journal of Clinical Nutrition, v. 11, n. 2, p. S77-S78, 2002.

IBGE - INSTITUTO BRASILEIRO DE GEOGRAFIA E ESTATÍSTICA. Censo 2000. Disponível em: <http://www.ibge. gov.br>. Acesso em: 20 jan. 2005.

LEVY, A. S.; FEIN, S. B. Consumer's ability to perform tasks using nutrition labels. Journal of Nutrition Education, v. 30, n. 4, p. 210-217, 1998.

LIMA, A; GUERRA, N. B.; LIRA, B. F. Evolução da legislação brasileira sobre rotulagem de alimentos e bebidas embalados e sua função educativa para promoção da saúde. Higiene Alimentar, v. 17, n. 110, p. 12-17, 2003.

MARIETTA, A. B; WELSHIMER, K. J.; ANDERSON, S. L. Knowledge, attitudes, and behaviors of college students regarding the 1990 nutrition labelling education act food labels. Journal of the American Dietetic Association, v. 99, n. 4, p. 445-449, 1999.

MATTA, I. E. A.; HENRIQUES, P.; SILVA, Y. Adequação à legislação vigente, da rotulagem de alimentos diet e light comercializados no Rio de Janeiro, RJ. Higiene Alimentar, v. 20, n. 147, p. 97-103, 2006.

MAPA - MINISTÉRIO DA AGRICULTURA PECUÁRIA E ABASTECIMENTO. Portaria no 371, de 04 de setembro de 1997. Estabelece o regulamento técnico para rotulagem de alimentos embalados. Disponível em: $<$ http://www.agricultura.gov.br $>$ Acesso em: 18 jul. 2003.

MINISTÉRIO DA SAÚDE. RESOLUÇÃO n 196 , de 10 de outubro de 1996. Aprovar diretrizes e normas regulamentadoras de pesquisas envolvendo seres humanos. Disponível em: <http://www.anvisa. gov.br>. Acesso em: 26 abr. 2005.
MIRILLI, J. C.; RESENDE, M. T. R.; MARTINELLI, M. A. Atualização sobre as atividades Codex Alimentarius. Higiene Alimentar, v. 10, n. 46, p. 8-9, 1996.

MONDINI, L; MONTEIRO, C. A. Mudanças no padrão de alimentação da população urbana brasileira: 1962-1988. Revista de Saúde Pública, v. 28, n. 6, p. 433-439, 1994.

NESTLE, M. et al. Behavioral and social influences on food choice. Nutrition Reviews, v. 56, n. 5, p. S50-S74, 1998.

NEUHOUSER, M.; KRISTAL, A. R.; PATTERSON, R. E. Use of food nutrition labels is associated with lower fat intake. Journal of the American Dietetic Association, v. 99, n. 1, p. 45-53, 1999.

NUNES, M. C. et al. Avaliação das sobremesas lácteas: Características que podem comprometer a garantia da qualidade. Higiene Alimentar, v. 12, n. 58, p. 41-48, 1998.

OMS - ORGANIZAÇÃO MUNDIAL DA SAÚDE. Obesity: preventing and managing the global epidemic. Working Group on Obesity. Geneva: World Health Organization, 1998.

PIPETONE, M. A. P. Educação para o consumo de alimentos. Higiene Alimentar, v. 19, n. 132, p. 18-23, 2005.

QUAST, D. Legislação e informação nutricional: o que o consumidor precisa saber? In: Novos aspectos de legislação sobre rotulagem de alimentos - funcionais, transgênicos, meio ambiente e comércio exterior. Campinas: Instituto de Tecnologia de Alimentos, abr. 2002. p. $47-50$.

ROBINSON, T. N. Does television viewing cause childhood obesity? JAMA, n. 279, p. 959, 1998.

SAUERBRONN, A. L. A. Análise laboratorial da composição de alimentos processados como contribuição ao estudo da rotulagem nutricional obrigatória de alimentos e bebidas embalados no Brasil. Rio de Janeiro, 2003. 69p. Dissertação - (Mestrado em Vigilância Sanitária), Instituto Nacional de Controle de Qualidade em Saúde, Fundação Oswaldo Cruz.

SHIMIZU, T. Newly established regulation in Japan: foods with health claims. Asia Pacific Journal of Clinical Nutrition, v. 11, n. 2, p. S94-S96, 2002.

SLOAN, A. E. What consumers want and don't want on food and beverage labels. Food Technology, v. 57, n. 11, p. 26-36, 2003.

TABAI, K. C. Análise do controle de alimentos no Brasil: da intervenção governamental à participação de consumidores e suas organizações. Higiene Alimentar, v. 16, n. 97, p. 22-25, 2002.

TEE, E. S. et al. Current status of nutrition labelling and claims in the South-East Asian region: are we in harmony? Asia Pacific Journal of Clinical Nutrition, v. 11, n. 2, p. S80-S86, 2002.

WOOD, R. A. Food manufacturing and the allergic consumer: Accidents waiting to happen. Journal Allergy of Clinical Immunology, v. 109, n. 6, p. 920-922, 2002.

ZARKIN, G. A. et al. Potential health benefits of nutrition label changes. American Journal of Public Health, v. 83, n. 5, p. 717-724, 1993. 